\title{
Reversible electrocardiographic changes in severe acute asthma
}

\author{
DAVID SIEGLER
}

From Brompton Hospital, London

Siegler, D. (1977). Thorax, 32, 328-332. Reversible electrocardiographic changes in severe acute asthma. Previous reports have documented the occurrence of reversible electrocardiographic changes including right axis deviation, $\mathbf{P}$ pulmonale, right bundle-branch block, and ST-segment and $\mathrm{T}$-wave abnormalities in patients with acute attacks of asthma. In a further systematic study, the electrocardiographs of 63 patients admitted with severe acute asthma have been evaluated. The most consistent change was an abnormally vertical P-wave axis in $78 \%$ of the patients. $\mathbf{P}$ pulmonale was present in $22 \%$ and right ventricular enlargement in only one patient. Right axis deviation, right bundle-branch block, and rhythm abnormality were not present in any patient. In $11 \%$, ST-segment or T-wave abnormalities suggesting myocardial ischaemia were noted. These abnormalities persisted for up to nine days and were unexplained. Other ECG abnormalities in acute asthma may reflect positional changes of the heart due to overdistension of the lungs. All ECG changes resolved after clinical improvement.

Reversible abnormalities in the electrocardiogram are well recognised in patients with acute attacks of bronchial asthma. Previously reported changes have included right axis deviation, prominent $\mathbf{P}$ waves suggesting right atrial enlargement, inversion of $T$ waves, right bundle-branch block, and abnormalities of the ST segment (Gunstone, 1971; Rebuck and Read, 1971; Da Costa and Chia, 1974). A further systematic study was prompted by observations which conflicted with previously published data. The electrocardiograms of 63 patients with severe acute asthma have been evaluated and attempts made to correlate ECG changes with physiological measurements of the severity of the attack.

\section{Material and methods}

The case records of all adult patients below the age of 50 years admitted to the medical intensive care ward of the Brompton Hospital between 1 January 1975 and 31 March 1976 with a confirmed diagnosis of status asthmaticus were studied. These were all patients known to have bronchial asthma for whom admission to hospital was necessitated by an acute attack of asthma failing to respond to their usual maintenance therapy. Patients were included in the study only if an ECG had been performed on the day of admission and thus within the first 24 hours (and usually within the first 12 hours) of their stay in hospital. Patients known to have coronary artery disease, valvular heart disease, or systemic hypertension were ex-음 cluded. Sixty-three patients were thus included in $\underset{x}{\otimes}$ the study, 34 female and 29 male, with an age $\frac{0}{\partial}$ range of 15 to 49 years and a mean age of 33 years. In each patient, on admission, plasma urea 8 and electrolytes, arterial blood gases, peak expiratory flow rate, forced expiratory volume in the $\frac{\text { ? }}{3}$ first second $\left(\mathrm{FEV}_{1}\right)$, and forced vital capacity (FVC) were estimated. The presence and magnitude of pulsus paradoxus were noted. A standard 12-lead ECG was performed. Where this was ab-o normal on admission, the ECG in most cases was $\tilde{O}$ repeated until it had returned to normal. The $\underset{\omega}{N}$ ECG was examined for abnormalities of rhythm $\sigma$ and of P-or QRS-wave axis. Right atrial enlarge-o ment was judged to be present if the height of the $P$ wave in standard leads II and III exceeded $2.5 \stackrel{\odot}{\rightarrow}$ mm (P pulmonale). Right ventricular hypertrophy was regarded as being present if there was right axis deviation to beyond $+110^{\circ}$, the height of the $\stackrel{\odot}{\stackrel{\Phi}{9}}$ $R$ wave in $V_{1}$ exceeded $5 \mathrm{~mm}$, and the ratio of $R-\stackrel{\mathbb{Q}}{\Omega}$ wave amplitude to $S$-wave amplitude in $V_{B}$ exceeded unity (Estes, 1974). The $P$-wave axis was abnormally vertical, ie, more than $90^{\circ}$, if the $\mathrm{P}$ 응 
wave in standard leads II and III was taller than in standard lead $I$ and the $P$ wave was negative in lead aVL (Estes, 1974).

\section{Results}

For the whole group of patients the mean arterial $\mathrm{PO}_{2} \pm 1 \mathrm{SD}$ on admission was $64 \mathrm{~mm} \pm 15 \mathrm{mmHg}$ $(8.5 \pm 2 \mathrm{kPa})$. Mean arterial $\mathrm{PCO}_{2}$ was $38 \mathrm{~mm} \pm$ $9 \mathrm{~mm} \mathrm{Hg}(5 \cdot 1 \pm 1 \cdot 2 \mathrm{kPa})$. Mean expiratory flow rate was $110 \pm 40$ litres per minute. Mean FEV $_{1}$ was $0.73 \pm 0.36$ litres and mean FVC $1.5 \pm 0.78$ litres.

The most consistent change was an abnormally vertical $\mathbf{P}$-wave axis in 48 of the patients $(78 \%)$. In those patients in whom further ECGs were performed once clinical improvement had been obtained, the P-wave axis had returned to normal, usually within three to four days (Figs 1 and 2). The next most common change was the appearance of $\mathbf{P}$ pulmonale in 12 patients $(22 \%)$, again reverting to normal in those patients who had further ECGs. Right ventricular enlargement was present in only one patient. Isolated right axis deviation was not seen in any patient. No rhythm disturbance was noted although a sinus tachycardia in excess of 100 per minute was invariable.

In seven patients $(11 \%)$ abnormalities of $T$ waves or ST segments were noted. In three of these, $T$-wave inversion across the chest leads from V1 to V5 was seen, and in one, electrical alternans of the QRS complexes was also present. In the other four subjects depression of the ST segments was noted: in three, this was present in leads II, III, and aVF, and in the remaining one in leads V3 to V6. The ST- and T-wave abnormalities were all reversible with clinical improvement. However, in three patients reversion to normal was slow, requiring two, four, and nine days respectively.

\section{Discussion}

The very high incidence of an abnormally vertical P-wave axis in acute asthma has not previously been described, although it is well documented as

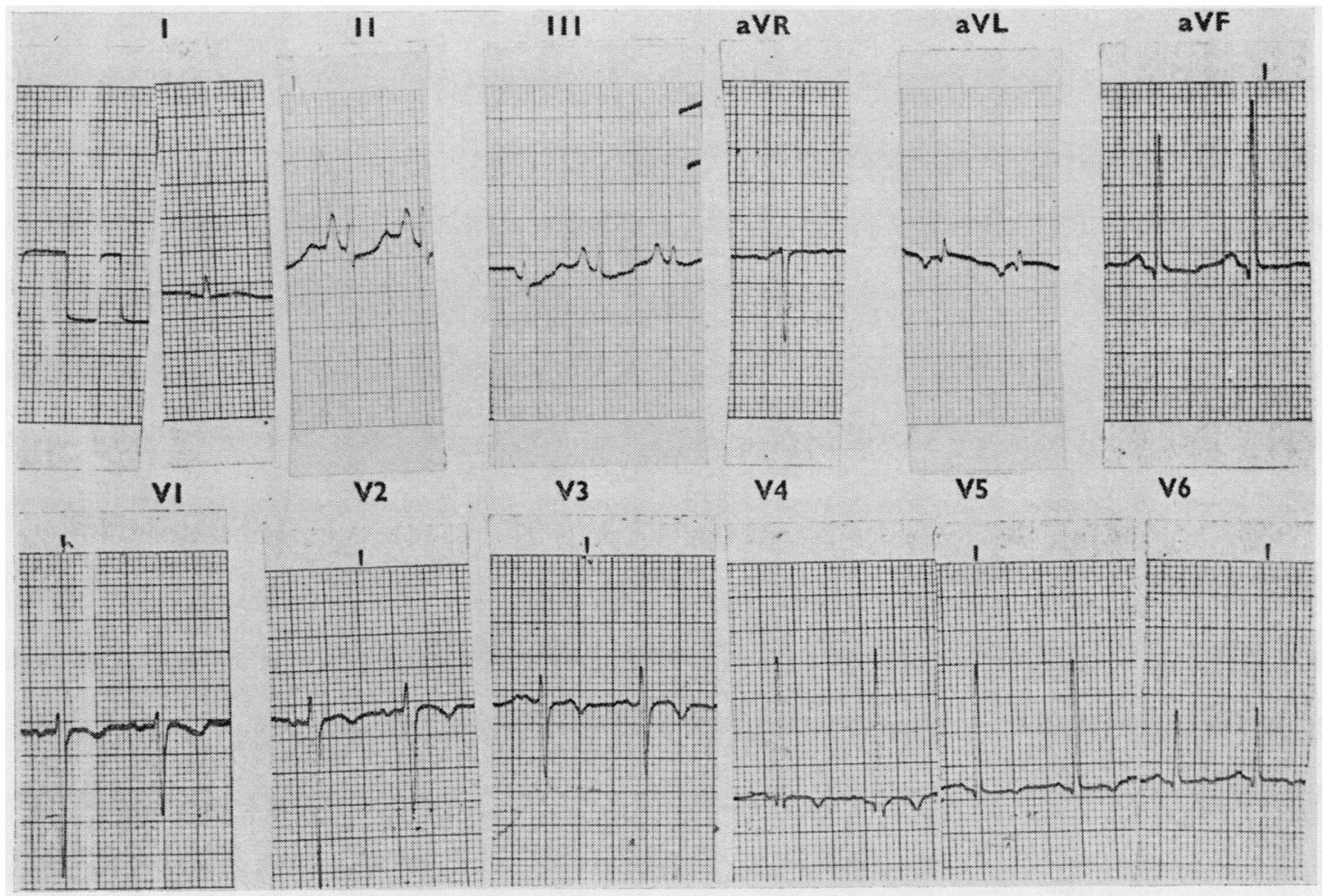

Fig. 1 Electrocardiogram of 29-year-old woman on the day of admission. Note P pulmonale, vertical $P$-wave axis, and $T$-wave inversion in anterior chest leads. 

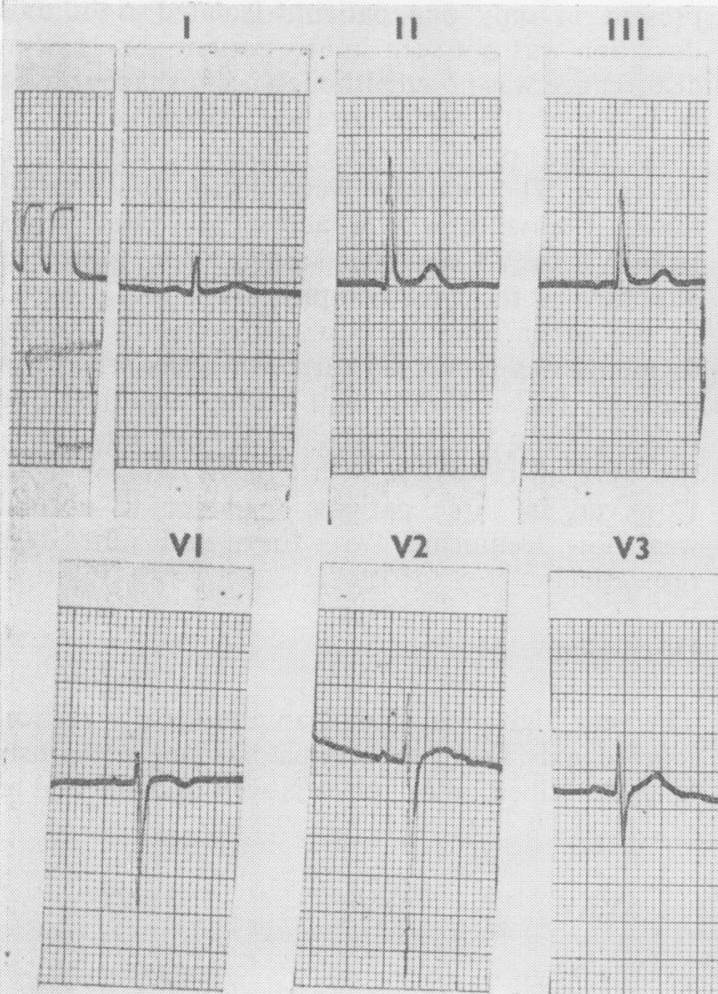

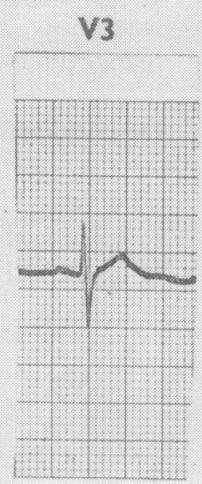

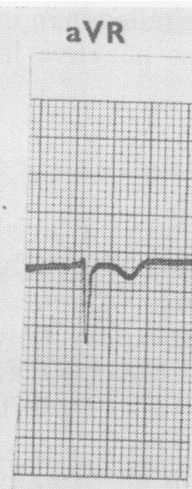



aVF
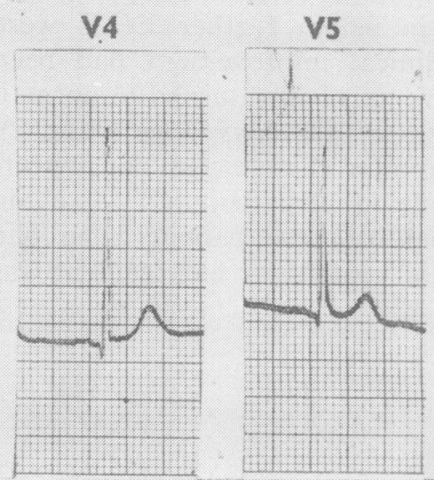

Fig. 2 Four days later ECG has reverted to normal.

an electrocardiographic manifestation of other forms of diffuse chronic airway obstruction. Chapman (1974) examined the ECG of a very large series of patients with diffuse airway obstruction referred for lung function testing. The series did not contain any patients with asthma. Chapman confirmed the finding of Spodick $(1959 \mathrm{a}, \mathrm{b})$ that a $\mathrm{P}$-wave axis of more than $90^{\circ}$ was the only distinctive ECG finding in these patients. He showed a good correlation between a vertical P-wave axis and the degree of airway obstruction as measured by the $\mathrm{FEV}_{1}$ as a percentage of $\mathrm{FVC}$, and less good correlations with impairment of the carbon monoxide transfer factor and the presence of hyperinflation of the lungs. The cause of the vertical $\mathbf{P}$-wave axis is not certain but probably reflects hyperinflation of the lungs leading to rotation of the heart to a more vertical position. It may be that for some reason the P-wave axis is a more sensitive indicator of this rotation than the QRS axis because in no patient in the present series was abnormally clockwise rotation of the QRS axis noted. Chapman (1974) also suggested that overinflated lungs conduct the ECG complexes to the anterior chest wall less well than normal lungs, and the normal conducting pathways to the chest $\stackrel{x}{x}$ wall are thus modified, leading to ECG abnormalities.

The next most common abnormality was the finding of $P$ pulmonale, suggesting right atrial en-

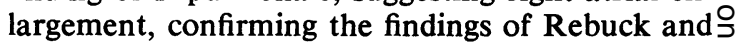
Read (1971) in 6 out of 76 patients and those of $D$ Da Costa and Chia (1974) in 8 of 18 patients. Gunstone (1971) performed right heart catheterisa- No tion in nine patients with acute asthma. In fiveof the patients the ECG showed such abnormalities as $\mathbf{P}$ pulmonale, right axis deviation, right bundle- $\omega$ branch block, and T-wave inversion. The pulmonary artery pressures were no higher in these patients than in the four remaining patients whose $\mathbb{\mathscr { D }}$ electrocardiograms were within normal limits. Schmock et al. (1971) attempted to correlate the ECG changes in a group of patients with chronic bronchitis and emphysema with postmortem $\stackrel{\odot}{\stackrel{\odot}{\odot}}$ examination of the lungs and heart. They found $\mathscr{\complement}$ that in all patients whose ECG demonstrated a $P$ O pulmonale, right ventricular enlargement was o present. In the absence of right ventricular en- 
largement, $\mathbf{P}$ pulmonale was absent. However, in the present series, in only one patient with $P$ pulmonale were there additional ECG criteria of right ventricular enlargement suggesting that in acute asthma $\mathbf{P}$ pulmonale per se is not a manifestation of right ventricular enlargement.

In the present study, the most unexpected and persistent ECG abnormalities were in those patients with $\mathrm{T}$-wave and ST-segment changes suggesting myocardial ischaemia. This confirms the finding of Rebuck and Read (1971), who noted similar abnormalities in 5 of their 76 patients and of Da Costa and Chia (1974) in 7 of their 18 patients. It is unlikely that these changes represent myocardial damage due to hypercapnia or hypoxaemia. The arterial $\mathrm{PCO}_{2}$ was normal in all seven subjects in the present series. In addition, the changes persisted long after any degree of hypoxaemia had been corrected, and in only two patients was the initial arterial $\mathrm{Po}_{2}$ below the mean value for the whole group of patients. It is of interest that Lary and Goldschlager (1974) described seven patients in whom exercise tests were being carried out to confirm a diagnosis of cardiac ischaemia and in whom hyperventilation led to 'false-positive' exercise electrocardiograms with the development of ST depression, elevation or flattening without the necessity for exercise. These authors noted that $\mathrm{T}$-wave inversion had previously been reported in similar circumstances. Respiratory alkalosis and abnormal potassium flux across myocardial cell membranes had already been excluded as causes of the apparent ischaemic changes (Barker et al., 1939; Biberman et al., 1971) and the authors concluded that the appearances could be due to autonomic nervous system activity or to adrenergic stimulation either therapeutically or by endogenous catecholamine release which might result in asynchronous repolarisation on the myocardium. Hyperventilation and endogenous or therapeutic sympathomimetic stimulation could certainly explain the appearances of ischaemia in the first few hours of an attack of asthma but will not account for the persistence of these changes for several days as was seen in the present study. The phenomenon remains unexplained. In these seven patients as a whole ECG changes of 'ischaemia' could not be correlated with differences in blood gases, spirometry or magnitude of pulses paradoxus from the group of patients as a whole. Indeed, it was not possible to correlate any of the ECG abnormalities with abnormalities of blood gases or spirometry. This confirms the previous findings of Rao et al. (1974) in 14 children with acute asthma in whom ECG changes did not consistently correlate with physiological abnormalities.

The inconsistency between the present series and previous reports was the absence from the present series of such changes as right axis deviation and right bundle-branch block. It could be argued that delays in recording the ECG could lead to these abnormalities being missed. This is a theoretical weakness of retrospective studies such as the present one. Rebuck and Read (1971) have pointed out that some ECG changes may disappear within hours. However, any delay in recording the ECG was unlikely to have made any material difference to the findings in the present study as some gross abnormalities did in fact persist for several days. The invariable persistence of sinus tachycardia suggests further that the heart was in some way still being influenced by the severe airway obstruction.

Finally, it is possible that all ECG changes except for those suggesting myocardial ischaemia are in fact explicable on purely anatomical grounds. Overdistension of the lungs, in addition to altering the electrical axes, may alter the position of the heart to such an extent that the anterior chest leads, for instance, no longer reflect the activity of that part of the heart which they would normally individually represent.

I thank Dr. T. J. H. Clark for helpful discussion and practical criticism of the manuscript.

\section{References}

Barker, P. S., Schrader, E. L., and Ronzoni, E. (1939). The effects of alkalosis and of acidosis upon the human electrocardiogram. American Heart Journal, 17, 169-186.

Biberman, L., Sarma, R. N., and Surawicz, B. (1971). T-wave abnormalities during hyperventilation and isoproterenol infusion. American Heart Journal, 81, 166-174.

Chapman, T. T. (1974). Lung function tests and a 'vertical' $P$ wave axis in the ECG. Thorax, 29, 106109.

Da Costa, J. L. and Chia, B. L. (1974). The electrocardiographic changes in bronchial asthma and their relationship to the severity of airways obstruction. Singapore Medical Journal, 15, 101-105.

Estes, E. H., Jr. (1974). The ventricular electrocardiogram. In The Heart, edited by J. W. Hurst et al., 3rd edition, p. 306. McGraw-Hill, New York.

Gunstone, R. F. (1971). Right heart pressures in bronchial asthma. Thorax, 26, 39-45.

Lary, D. and Goldschlager, N. (1974). ECG changes during hyperventilation resembling myocardial ischemia in patients with normal coronary arteriograms. American Heart Journal, 87, 383-390. 
Rao, M., Steiner, P., Paydat, R., Padre, R., and Torres, R. R. (1974). Electrophysiological changes in the heart of children with bronchial asthma during the acute attack and later during remission. Journal of Asthma Research, 11, 113-121.

Rebuck, A. S. and Read, J. (1971). Assessment and management of severe asthma. American Journal of Medicine, 51, 788-798.

Schmock, C. L., Mitchell, R. S., Pomerantz, B., Pryor, R., and Maisel, C. L. (1971). The electrocardiogram in chronic airways obstruction: the role of bronchitis and emphysema. Chest, 60, 335-340.
Spodick, D. H. (1959a). Electrocardiographic studies $\stackrel{\overrightarrow{\vec{F}}}{\stackrel{\vec{H}}{+}}$ in pulmonary disease I. Electrocardiographic abnor-O malities in diffuse lung disease. Circulation, 20, 1067-1072.

Spodick, D. H. (1959b). Electrocardiographic studies in pulmonary disease II. Establishment of criteria for the E.C.G. inference of diffuse lung disease.œ Circulation, 20, 1073-1074.

Requests for reprints to: Dr. D. Siegler, Senior $\vec{\omega}$ Medical Registrar, Brompton Hospital, Fulham Road, London SW3 6HP. 